WU B 01-03

DESY-01-118

hep-ph/0108220

August 2001

\title{
The annihilation of virtual photons into pseudoscalar mesons
}

\author{
M. Diehl円 \\ Deutsches Elektronen-Synchrotron DESY, 22603 Hamburg, Germany \\ P. Krolle and C. Vogt \\ Fachbereich Physik, Universität Wuppertal, 42097 Wuppertal, Germany
}

\begin{abstract}
We investigate the possibility to constrain the pion distribution amplitude from the $\gamma^{*} \gamma^{*} \rightarrow \pi$ transition. For a surprisingly large range in the two photon virtualities we find that the transition form factor is essentially independent of the distribution amplitude. This in turn entails a parameter-free prediction of QCD. The $\gamma^{*} \gamma^{*} \rightarrow \eta, \eta^{\prime}$ form factors are also briefly discussed. We estimate that experimental studies might be feasible at the existing $e^{+} e^{-}$experiments BaBar, Belle, and CLEO.
\end{abstract}

\footnotetext{
${ }^{1}$ Email: markus.diehl@desy.de

${ }^{2}$ Email: kroll@theorie.physik.uni-wuppertal.de

${ }^{3}$ Email: cvogt@theorie.physik.uni-wuppertal.de
} 


\section{Introduction}

One of the simplest exclusive observables is the form factor $F_{P \gamma^{(*)}}$ for transitions from a real or virtual photon to a pseudoscalar meson $P$. Its behavior at large momentum transfer is determined by the expansion of a product of two electromagnetic currents about light-like distances [1]. The form factor then factorizes into a hard scattering amplitude, which is known including the first-order perturbative QCD corrections [2, 3], and a soft matrix element, parameterized by a process independent meson distribution amplitude $\Phi(\xi)$.

For space-like momentum transfer the form factors $F_{P \gamma^{(*)}}$ can be accessed in $e^{+} e^{-} \rightarrow e^{+} e^{-} P$. The measurement by CLEO [4] for quasi-real photons and $P=\pi, \eta, \eta^{\prime}$ has renewed the interest in these quantities, and many papers have been devoted to their theoretical analysis, e.g. [5][13], to name a few. The CLEO data are consistent with distribution amplitudes of the pion, the $\eta$, and the $\eta^{\prime}$ which are rather close to the asymptotic form,

$$
\Phi_{\mathrm{AS}}(\xi)=\frac{3}{2}\left(1-\xi^{2}\right)
$$

where $\xi=2 x-1$, and $x$ is the usual momentum fraction carried by the quark inside the meson.

The purpose of the present article is to investigate the information contained in the form factors for $\gamma^{*} \gamma^{*} \rightarrow P$ transitions, beyond what we have already learned from the real-photon case. In Sect. 2 we cast the leading twist, next-to-leading order result for the $\gamma^{*} \gamma^{*} \rightarrow \pi$ form factor in a form useful for the purpose of our study. In Sect. 3 we attempt a critical appraisal of what we can and what we cannot deduce from the existing data on the transition $\gamma^{*} \gamma \rightarrow \pi$. The following two sections explore the $\gamma^{*}-\pi$ transition form factor in two different kinematical regimes. In Sect. 6 we briefly point out the specifics of the transitions to $\eta$ and $\eta^{\prime}$ mesons. Estimates of cross sections at the running experiments BaBar, Belle, and CLEO are given in Sect. 8, and we conclude in Sect. 8. Some technical details concerning the $\alpha_{s}$ corrections to $F_{\pi \gamma^{*}}$ are given in an appendix.

\section{The $\gamma^{*}-\pi$ transition form factor to leading twist}

Let us begin with the discussion of the $\gamma^{*} \gamma^{*} \rightarrow \pi$ form factor. The $\gamma^{*} \gamma^{*} \pi$ vertex is parameterized by

$$
\Gamma_{\mu \nu}=-i e^{2} F_{\pi \gamma^{*}}(\bar{Q}, \omega) \varepsilon_{\mu \nu \alpha \beta} q^{\alpha} q^{\prime \beta},
$$

where we use the convention $\epsilon_{0123}=1$. Here $q$ and $q^{\prime}$ respectively denote the photon momenta corresponding to the Lorentz indices $\mu$ and $\nu$. We introduce the spacelike photon virtualities $Q^{2}=-q^{2}, Q^{\prime 2}=-q^{\prime 2}$, as well as

$$
\bar{Q}^{2}=\frac{1}{2}\left(Q^{2}+Q^{\prime 2}\right), \quad \omega=\frac{Q^{2}-Q^{\prime 2}}{Q^{2}+Q^{\prime 2}} .
$$

The values of $\omega$ range from -1 to 1 , but due to Bose symmetry the transition form factor is symmetric in this variable: $F_{\pi \gamma^{*}}(\bar{Q}, \omega)=F_{\pi \gamma^{*}}(\bar{Q},-\omega)$.

To leading-twist accuracy, i.e., in the collinear approximation and using only the valence Fock state of the pion, the transition form factor $F_{\pi \gamma^{*}}$ reads [2, 3]

$$
F_{\pi \gamma^{*}}(\bar{Q}, \omega)=\frac{f_{\pi}}{3 \sqrt{2} \bar{Q}^{2}} \int_{-1}^{1} d \xi \frac{\Phi_{\pi}\left(\xi, \mu_{F}\right)}{1-\xi^{2} \omega^{2}}\left[1+\frac{\alpha_{s}\left(\mu_{R}\right)}{\pi} \mathcal{K}\left(\omega, \xi, \bar{Q} / \mu_{F}\right)\right] .
$$




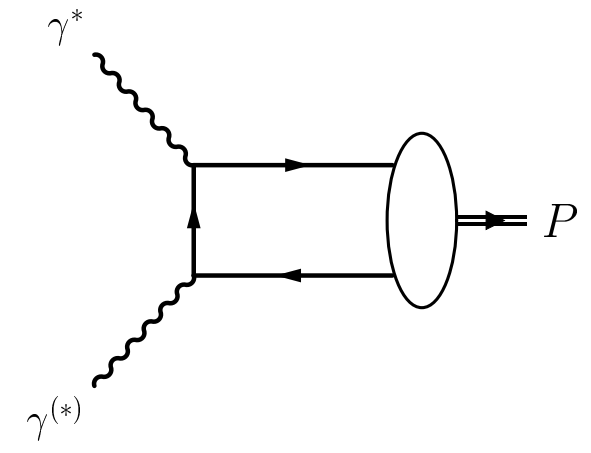

Figure 1: Lowest order Feynman graph for the $\gamma^{*} \gamma^{(*)} \rightarrow \pi$ transition. A second graph is obtained by interchanging the photon vertices.

The Feynman graphs contributing to leading order (LO) are shown in Fig. 1, and the next-to leading order (NLO) kernel $\mathcal{K}\left(\omega, \xi, \bar{Q} / \mu_{F}\right)$ in the $\overline{\mathrm{MS}}$ scheme is given in the appendix. $\mu_{F}$ and $\mu_{R}$ respectively denote the factorization and renormalization scales, both to be taken of order $\bar{Q}$, and $f_{\pi} \approx 131 \mathrm{MeV}$ is the well-known pion decay constant. $\Phi_{\pi}$ is the pion distribution amplitude, which we expand upon Gegenbauer polynomials $C_{n}^{3 / 2}(\xi)$, the eigenfunctions of the leading-order evolution kernel for mesons [1],

$$
\Phi_{\pi}\left(\xi, \mu_{F}\right)=\Phi_{\mathrm{AS}}(\xi)\left[1+\sum_{n=2,4, \ldots}^{\infty} B_{n}\left(\mu_{F}\right) C_{n}^{3 / 2}(\xi)\right] .
$$

The Gegenbauer coefficients are scale dependent, to LO they evolve according to

$$
B_{n}\left(\mu_{F}\right)=B_{n}\left(\mu_{0}\right)\left(\frac{\alpha_{s}\left(\mu_{F}\right)}{\alpha_{s}\left(\mu_{0}\right)}\right)^{\gamma_{n} / \beta_{0}},
$$

where $\mu_{0}$ is the starting scale of evolution and $\beta_{0}=11-2 n_{f} / 3$. The anomalous dimensions $\gamma_{n}$ are positive numbers increasing with $n$, so that for $\ln \mu_{F} \rightarrow \infty$ any distribution amplitude evolves into $\Phi_{\mathrm{AS}}$ with higher order terms in (5) gradually becoming suppressed. Hence, the limiting behavior of the transition form factor for real photons is

$$
F_{\pi \gamma}(\bar{Q}, \omega= \pm 1) \longrightarrow \frac{f_{\pi}}{\sqrt{2} \bar{Q}^{2}},
$$

which is a parameter-free QCD prediction once $f_{\pi}$ is known. Note, however, that this limit is only approached logarithmically, and that the anomalous dimensions are not very large at small $n$. For $n_{f}=4$ flavors one has $\gamma_{2} / \beta_{0}=2 / 3, \gamma_{4} / \beta_{0} \approx 0.97, \gamma_{6} / \beta_{0} \approx 1.17$. As $n$ becomes large the $\gamma_{n}$ grow logarithmically, $\gamma_{n} \sim \frac{16}{3} \ln (n+1)$ being a good approximation already for $n=2$.

To NLO accuracy, the $C_{n}^{3 / 2}(\xi)$ are no longer eigenfunctions of the evolution, so that their coefficients do no evolve independently. Namely, $B_{n}\left(\mu_{F}\right)$ at $\mu_{F}>\mu_{0}$ depends on all coefficients $B_{2}\left(\mu_{0}\right), \ldots, B_{n}\left(\mu_{0}\right)$. NLO evolution resums logarithms $\alpha_{s}^{2} \log \left(\mu_{F} / \mu_{0}\right)$, compared with $\alpha_{s} \log \left(\mu_{F} / \mu_{0}\right)$ in LO evolution, and its effects will be more important when one evolves over a large interval in $\mu_{F}$ or when $\alpha_{s}$ at the starting scale $\mu_{0}$ is large. 
Using the Gegenbauer expansion (5), the integral in (丑) can be worked out analytically order by order in $n$, provided that $\mu_{R}$ is chosen to be independent of $\xi$. This results in

$$
F_{\pi \gamma^{*}}(\bar{Q}, \omega)=\frac{f_{\pi}}{\sqrt{2} \bar{Q}^{2}}\left[c_{0}\left(\omega, \mu_{R}\right)+\sum_{n=2,4, \ldots} c_{n}\left(\omega, \mu_{R}, \bar{Q} / \mu_{F}\right) B_{n}\left(\mu_{F}\right)\right],
$$

where the lowest-order coefficients $c_{n}$ read

$$
\begin{aligned}
c_{0}= & \frac{1}{\omega^{2}}\left[1-\left(1-\omega^{2}\right) \frac{\operatorname{artanh} \omega}{\omega}\right]-\frac{\alpha_{s}\left(\mu_{R}\right)}{\pi} \frac{1}{9 \omega^{2}}\left[15-\left(1-\omega^{2}\right)\left(15+4 \operatorname{artanh}^{2} \omega\right) \frac{\operatorname{artanh} \omega}{\omega}\right], \\
c_{2}= & \frac{1}{2 \omega^{4}}\left[15-13 \omega^{2}-\left(5-6 \omega^{2}+\omega^{4}\right) \frac{3 \operatorname{artanh} \omega}{\omega}\right]+\frac{\alpha_{s}\left(\mu_{R}\right)}{\pi} \mathcal{K}_{2}\left(\omega, \bar{Q} / \mu_{F}\right) \\
c_{4}= & \frac{1}{8 \omega^{6}}\left[315-420 \omega^{2}+113 \omega^{4}-\left(21-35 \omega^{2}+15 \omega^{4}-\omega^{6}\right) \frac{15 \operatorname{artanh} \omega}{\omega}\right] \\
& +\frac{\alpha_{s}\left(\mu_{R}\right)}{\pi} \mathcal{K}_{4}\left(\omega, \bar{Q} / \mu_{F}\right) .
\end{aligned}
$$

The analytical expressions of the $\alpha_{s}$ corrections $\mathcal{K}_{2}$ and $\mathcal{K}_{4}$ are rather lengthy and we refrain from showing them explicitly. Their dependence on $\ln \left(\bar{Q} / \mu_{F}\right)$ is partially compensated in $F_{\pi \gamma^{*}}(\bar{Q}, \omega)$ by the $\mu_{F}$ dependence of the $B_{n}\left(\mu_{F}\right)$. Notice that no such compensation takes place for the $\mu_{R}$ dependence to the order in $\alpha_{s}$ we are working in. Unless stated otherwise we will in the following take $\mu_{F}=\mu_{R}=\bar{Q}$, which is the virtuality of the quark propagators in Fig. 1 at $\xi=0$. The coefficients $c_{n}$ then depend weakly on $\bar{Q}$ via $\alpha_{s}(\bar{Q})$. Other scale choices lead, as usual, to results differing by terms of $\mathcal{O}\left(\alpha_{s}^{2}\right)$, which is beyond the accuracy of our analysis. We finally remark that for fixed $\omega$ the $F_{\pi \gamma^{*}}$ form factor only falls off like $\bar{Q}^{-2}$ at large $\bar{Q}$, in contrast to the $Q^{-2} Q^{\prime-2} \propto \bar{Q}^{-4}$ behavior of the vector meson dominance model [14].

In order to visualize the sensitivity of the form factor to the Gegenbauer coefficients we plot the lowest coefficients $c_{n}(\omega)$ in Fig. 2. Here and in the following we use the two-loop expression of $\alpha_{s}$ for $n_{f}=4$ flavors and $\Lambda_{\overline{\mathrm{MS}}}^{(4)}=305 \mathrm{MeV}$ [15]. We see a surprising behavior of the coefficients in $\omega$. In the real-photon limit $\omega \rightarrow 1$ the form factor is sensitive to all Gegenbauer moments with approximately equal weight, $c_{n}(\omega=1) \approx 1$. As soon however as one departs from this limit, the coefficients $c_{n}(\omega)$ decrease and become ordered as $c_{0}>c_{2}>c_{4}>\ldots$ Except for $c_{0}$ this decrease is rather fast. If $\omega<0.8$, for instance, the second coefficient $c_{2}$ is less than $40 \%$ of $c_{0}$. For a wide range of $\omega$, the form factor is essentially independent of the $B_{n}$, unless

they are unexpectedly large. The discussion of what can be learned from the measurement of $F_{\pi \gamma^{*}}$ naturally falls into two parts, concerning the kinematic regions $Q^{\prime 2} \ll Q^{2}$ and $Q^{\prime 2} \sim Q^{2}$, respectively.

\section{The real-photon limit}

Before embarking on the study of $F_{\pi \gamma^{*}}$ let us discuss some issues in the analysis of the realphoton limit. At $\omega=1$ one has

$$
c_{n}(\omega=1)=1+\frac{\alpha_{s}\left(\mu_{R}\right)}{\pi} \mathcal{K}_{n}\left(\omega=1, \bar{Q} / \mu_{F}\right)
$$



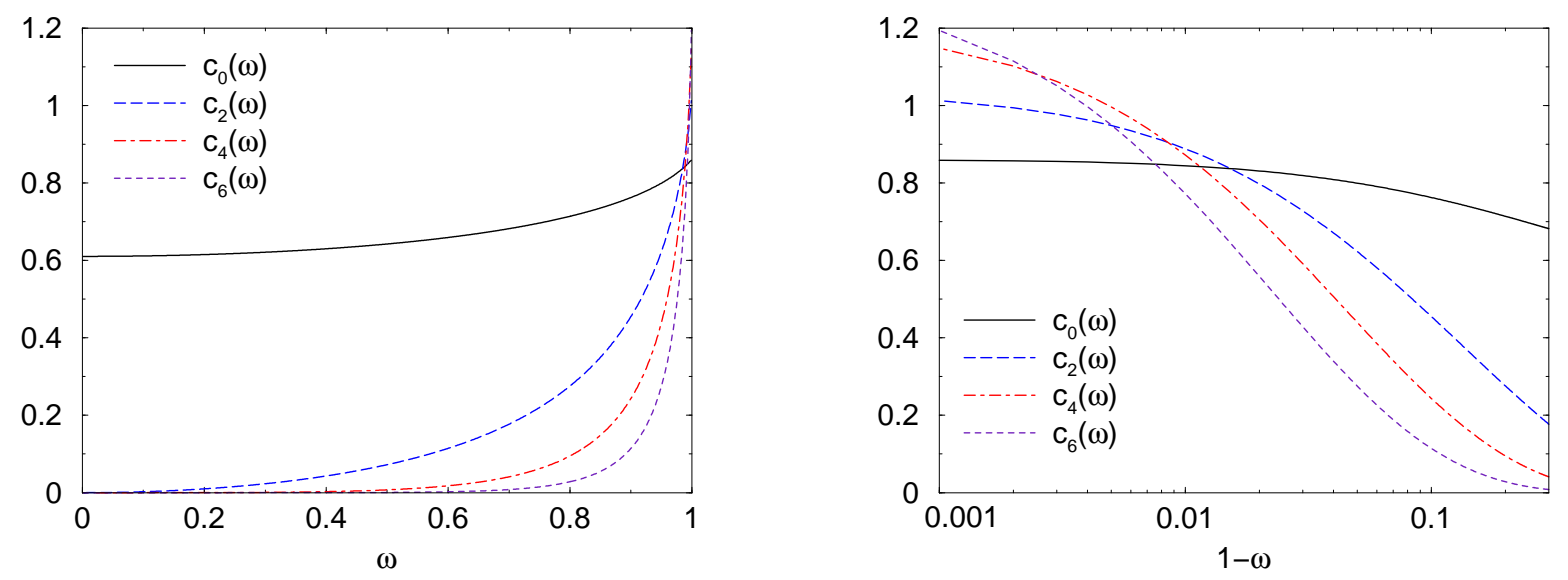

Figure 2: The coefficients $c_{n}(\omega)$ in the expansion (8) of the $\gamma^{*}-\pi$ transition form factor. NLO corrections are included with $\mu_{F}=\mu_{R}=\bar{Q}$, which is taken as $2 \mathrm{GeV}$.

where the first few coefficients explicitly read

$$
\mathcal{K}_{0}=-\frac{5}{3}, \quad \mathcal{K}_{2}=\frac{5}{3}\left(\frac{59}{72}-\frac{5}{6} \ln \frac{2 \bar{Q}^{2}}{\mu_{F}^{2}}\right), \quad \mathcal{K}_{4}=\frac{5}{3}\left(\frac{10487}{4500}-\frac{91}{75} \ln \frac{2 \bar{Q}^{2}}{\mu_{F}^{2}}\right) .
$$

The $\gamma-\pi$ transition form factor thus approximately probes the sum $1+\sum_{n} B_{n}$ of Gegenbauer coefficients. Due to evolution and the running of $\alpha_{s}$ the relative weights of the $B_{n}\left(\mu_{0}\right)$ in $F_{\pi \gamma}$ vary with $Q$, but only logarithmically. Extracting information on the Gegenbauer coefficients beyond their sum hence requires analyzing the form factor in a sufficiently large range of $Q$, say $Q_{\min } \leq Q \leq Q_{\max }$. While $Q_{\max }$ is usually set by the available data, the choice of $Q_{\min }$ is an important source of theoretical uncertainty, since at lower $Q$ power corrections to the leading twist result can become increasingly important. There are various approaches to describe such power corrections, all of which involve further assumptions or parameters.

A different source of uncertainty is that in practice analyses of $F_{\pi \gamma}$ data are performed with a truncation of the Gegenbauer series, setting all $B_{n}=0$ for $n \geq n_{0}$ with some $n_{0}$. This may be seen as an analog to the determination of parton distributions, where one typically chooses a functional form of the parton distributions at the starting scale of evolution and then fits its parameters to data on inclusive processes. Notice that setting $B_{n}$ to zero for all values of $\mu_{F}$ is strictly speaking not consistent with NLO evolution, which generates nonzero $B_{n}\left(\mu_{F}\right)$ even if $B_{n}\left(\mu_{0}\right)=0$. Müller [16] has shown that this can lead to important effects on the shape of the distribution amplitude, especially in the endpoint regions and when $\alpha_{s}$ at the starting scale is large. In the analysis [7] of the CLEO data, the impact of NLO evolution on the quantity $F_{\pi \gamma}$ was however found to be small compared with the NLO corrections to the hard scattering kernel. In the numerical studies of the present work we will be concerned with values of $\mu_{F}=\bar{Q}$ between 1 and $2 \mathrm{GeV}$. We choose $\mu_{0}=1 \mathrm{GeV}$ as the starting scale of evolution and take the LO formula (6), using however the two-loop expression of $\alpha_{s}$. Since the purpose of this paper is not a precise determination of $\Phi_{\pi}$, this should be sufficiently accurate. At the same time it keeps the analysis procedure simple as it allows us to work with a finite number of Gegenbauer coefficients at all scales.

The simplest analyses of the $F_{\pi \gamma}$ data assume $n_{0}=4$. This leads to stable results on $B_{2}$, although its value is subject to the uncertainties just discussed. Thus, for instance, Ref. [7] 
obtained the value $B_{2}\left(\mu_{0}=1 \mathrm{GeV}\right)=-0.15 \pm 0.04$ to NLO accuracy in the $\overline{\mathrm{MS}}$ scheme, using $\mu_{F}=\mu_{R}=Q$ and $Q_{\min }^{2}=3 \mathrm{GeV}^{2}$. Changing to $\mu_{F}=\mu_{R}=\bar{Q}$ as we prefer here, one finds $B_{2}\left(\mu_{0}=1 \mathrm{GeV}\right)=-0.06 \pm 0.03$ for both $Q_{\min }^{2}=2 \mathrm{GeV}^{2}$ and $3 \mathrm{GeV}^{2}$ within errors. This shift in the value of $B_{2}$ may be taken as an indication of the uncertainties due to uncalculated higher orders in the perturbative expansion. Melić et al. [13] have calculated the part of the $\mathcal{O}\left(\alpha_{s}^{2}\right)$ corrections that allows one to determine the BLM scale for $F_{\pi \gamma}$. Taking the distribution amplitude $\Phi_{\text {AS }}$ they find $\mu_{R} \approx Q / 3$ in the $\overline{\mathrm{MS}}$ scheme. The corresponding NLO prediction for $F_{\pi \gamma}$ is slightly below the CLEO data, in contrast to the one for $\Phi_{\mathrm{AS}}$ and $\mu_{R}=Q$, which is slightly above. Brodsky et al. [11] find consistency with the CLEO data when taking $\Phi_{\mathrm{AS}}$ and a yet lower renormalization scale together with a prescription for the saturation of $\alpha_{s}$, thus including effects beyond a leading twist perturbative analysis.

If one allows for $B_{2}$ and $B_{4}$ in the analysis there is no unique result for the individual coefficients. Rather there is a linear correlation between $B_{2}$ and $B_{4}$, which using (6), (8), (11) one can approximate as

$$
\begin{aligned}
f(\bar{Q}) & =B_{2}\left(\mu_{0}\right)+\left(1+\frac{\alpha_{s}\left(\mu_{R}\right)}{\pi}\left(\mathcal{K}_{4}-\mathcal{K}_{2}\right)\right)\left(\frac{\alpha_{s}\left(\mu_{F}\right)}{\alpha_{s}\left(\mu_{0}\right)}\right)^{\left(\gamma_{4}-\gamma_{2}\right) / \beta_{0}} B_{4}\left(\mu_{0}\right)+\mathcal{O}\left(\alpha_{s}^{2}\right) \\
& \approx B_{2}\left(\mu_{0}\right)+\left(1+0.66 \alpha_{s}(\bar{Q})\right)\left(\frac{\alpha_{s}(\bar{Q})}{\alpha_{s}\left(\mu_{0}\right)}\right)^{0.3} B_{4}\left(\mu_{0}\right)+\mathcal{O}\left(\alpha_{s}^{2}\right),
\end{aligned}
$$

where we have taken $\mu_{F}=\mu_{R}=\bar{Q}$ and $n_{f}=4$ when going from the first to the second line. The function $f(\bar{Q})$ includes the data on $F_{\pi \gamma}$ and the term with $c_{0}$ in (8). It may also absorb possible power corrections as far as they are independent of $\Phi_{\pi}$. Only the mild logarithmic $Q$ dependence due to evolution and the running of $\alpha_{s}$ restricts the allowed values of $B_{2}$ and $B_{4}$ to a finite region in parameter space. The factor multiplying $B_{4}\left(\mu_{0}\right)$ in (12) varies between 1.31 and 1.05 for $\bar{Q}^{2}$ between 1 and $4 \mathrm{GeV}^{2}$, the latter corresponding to the center of the highest $Q^{2}$ bin in the CLEO measurement.

The experimental errors of the form factor data allow deviations from the linear correlation (12). This correlation is nicely illustrated by the $\chi^{2}$-contour plots in Fig. 3, generated by MINUIT for our leading-twist NLO analysis. Comparison of the plots reveals that for $Q_{m i n}^{2}=3 \mathrm{GeV}^{2}$ the allowed parameter regions are enlarged since both the number of data points included in the fit and the lever arm in $Q$ are smaller. On the other hand, power corrections should be less important in this case. We also observe from the figure that the $1 \sigma$ range for $B_{2}$ and $B_{4}$ obtained from the fit with $Q_{\min }^{2}=2 \mathrm{GeV}^{2}$ is embedded in that for $Q_{\min }^{2}=3 \mathrm{GeV}^{2}$, indicating that the fits are consistent with each other. Within the experimental errors, logarithmic effects suffice to describe the residual $Q^{2}$ dependence of the CLEO data for $Q^{2} F_{\pi \gamma}\left(Q^{2}\right)$ above $2 \mathrm{GeV}^{2}$. We emphasize that this finding does not prove that power corrections are indeed small in that region, it rather illustrates the difficulty to distinguish a power from a logarithmic behavior in $Q^{2}$ with data in the range between 2 and $8 \mathrm{GeV}^{2}$.

From the above exercise we conclude that only extreme values of $\left|B_{2}\right|$ and $\left|B_{4}\right|$, say above 1 or 2 , are ruled out if higher order Gegenbauer coefficients are neglected. A compact way of presenting the result of the fit with two free coefficients is to use the linear combinations $B_{2}+B_{4}$ and $B_{2}-B_{4}$, which have approximately uncorrelated errors. Taking $Q_{\min }^{2}=2 \mathrm{GeV}^{2}$ we obtain $B_{2}+B_{4}=-0.06 \pm 0.08$ and $B_{2}-B_{4}=0.0 \pm 0.9$ at $\mu_{0}=1 \mathrm{GeV}$. This illustrates that, within a leading twist NLO analysis, the CLEO data on the $\gamma^{*} \gamma \rightarrow \pi$ form factor is 

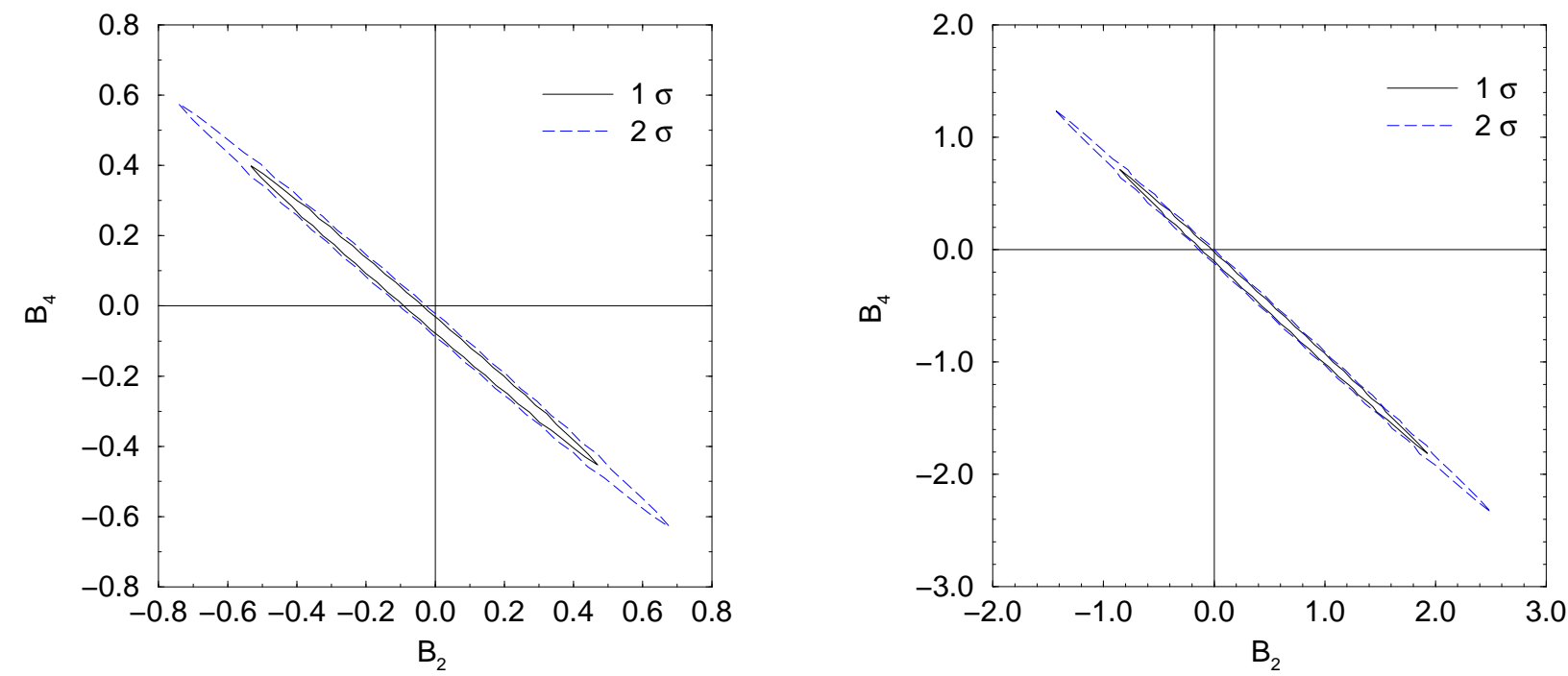

Figure 3: $1 \sigma$ and $2 \sigma \chi^{2}$-contour plots for a two parameter fit to the CLEO data on $F_{\pi \gamma}$ choosing $Q_{\text {min }}^{2}=2 \mathrm{GeV}^{2}$ (left) and $3 \mathrm{GeV}^{2}$ (right). Values of the Gegenbauer coefficients $B_{2}$ and $B_{4}$ refer to a factorization scale of $\mu_{0}=1 \mathrm{GeV}$. The data is fitted to the leading twist NLO expression (4) with $\mu_{F}=\mu_{R}=Q / \sqrt{2}$. The $\chi^{2}$ is calculated from the errors on the data and does not include a theory error.

insufficient for an unambiguous determination of the pion distribution amplitude, rather it approximately fixes the sum $\sum_{n} B_{n}$ to be close to zero. We cannot decide from the existing data on $F_{\pi \gamma}$ whether the small value of the sum $\sum_{n} B_{n}$ results from the cancellation of rather large individual terms or from the smallness of the $B_{n}$ themselves. Additional information from processes where $\Phi_{\pi}$ enters in a different way, would be highly welcome to settle this issue. One candidate are the decays of charmonium states into pion pairs, where however the quality of the data as well as unsuppressed color octet contributions [17] do not permit a conclusive analysis at present. Many theoretical studies have been devoted to the elastic pion form factor, cf. e.g. [11, 18, 19, 20, 21], but data at large $Q^{2}$ is scarce. A different way to probe the pion wave function is provided by diffractive dissociation of a pion into jets [22]. It is however not clear at present to which precision information on $\Phi_{\pi}$ can be obtained in that process [23].

We will investigate below what data on the $\gamma^{*}-\pi$ transition form factor could contribute, but before this we should discuss the question of theoretical uncertainties in $F_{\pi \gamma}$. The $\chi^{2}$ contours in Fig. 3 only reflect the errors on the form factor data. We have already mentioned the uncertainty from the choice of factorization and renormalization scales. Much harder to estimate is the role of power corrections. Contributions from various dynamical sources have been studied in the literature, such as higher twist distribution amplitudes [24, 25], the nonperturbative behavior of $\alpha_{s}(\mu)$ in the infrared region [11, 19], or transverse momentum effects in the hard scattering subprocess [5, 7, 10].

At this point we wish to comment on the analysis of the CLEO data presented in [26]. Power corrections due to the hadronic component of the photon have been modeled there within QCD sum rules. The relative weights of $B_{2}\left(\mu_{0}\right)$ and $B_{4}\left(\mu_{0}\right)$ in $F_{\pi \gamma}$ then display a much stronger $Q^{2}$ dependence than in the leading twist case (12), which leads to a much smaller allowed parameter region than in our Fig. 3. A large part of the deviation between 
the $F_{\pi \gamma}$ data and the result obtained with $\Phi_{\mathrm{AS}}$ in that analysis is due to the inclusion of twist-four distribution amplitudes. Their shape is taken to be the asymptotic one, and for their normalization results from QCD sum rule calculations as given in [24] are used. No error is however assigned to this input in the analysis. Note that an uncertainty of about $\pm 30 \%$ has been estimated for the relevant normalization constant $\delta^{2}$ in [27. Given this and the fact that only certain power corrections are taken into account in the QCD sum rule technique, we feel that the errors on the extracted Gegenbauer coefficients given in 26] are subject to a significant model dependence.

Several of the analyses cited above find moderate but non-negligible power corrections to $F_{\pi \gamma}$ in the $Q^{2}$ range where most of the CLEO data is concentrated. We find that our limited ability to reliably describe, let alone to calculate power corrections precludes us from drawing firm quantitative conclusions and from discriminating between many of the available theory predictions for $\Phi_{\pi}$. The best remedy to this situation we can see is data on $F_{\pi \gamma}$ at the highest possible $Q^{2}$. Given the luminosity of the presently running experiments BaBar, Belle and CLEO, a substantial improvement should be possible over the statistics of the available data [由], which is based on an integrated luminosity of about $3 \mathrm{fb}^{-1}$.

Notice that the range of theory predictions on $\Phi_{\pi}$ is considerable. For instance, the QCD sum rule analysis of Braun and Filyanov 28] gave $B_{2}(1 \mathrm{GeV})=0.44$ and $B_{4}(1 \mathrm{GeV})=0.25$ under the assumption that higher order coefficients are negligible. On the other side, a still preliminary result from lattice QCD [29] gives $B_{2}=-0.41 \pm 0.06$ at a low scale. Between these extremes many studies, using e.g. light-cone QCD sum rules with non-local condensates [30], the transverse lattice [31], or the instanton model of QCD [32] obtain a distribution amplitude either slightly broader or slightly narrower than $\Phi_{\mathrm{AS}}$.

\section{The region $Q^{\prime 2} \ll Q^{2}$}

With the lessons from the real-photon limit in mind let us now investigate the region where one of the photons is slightly off-shell. In order to gain some insight in the importance of power corrections we estimate transverse momentum effects by employing the modified perturbative approach [33, 20]. It has been applied to the case of $\gamma^{*}-\pi$ transitions in [5, 7], and to the case at hand in [6]. In this approach the expression (4) is replaced by

$$
F_{\pi \gamma^{*}}(\bar{Q}, \omega)=\frac{1}{4 \sqrt{3} \pi^{2}} \int d \xi d^{2} \mathbf{b} \hat{\Psi}_{\pi}^{*}\left(\xi,-\mathbf{b}, \mu_{F}\right) K_{0}(\sqrt{1+\xi \omega} \bar{Q} b) \exp \left[-S\left(\xi, b, \bar{Q}, \mu_{R}\right)\right]
$$

where $K_{0}$ is the modified Bessel function of order zero, representing the Fourier transform of the leading-order hard scattering amplitude in momentum space. The quark-antiquark separation $\mathbf{b}$ is canonically conjugated to the usual transverse momentum $\mathbf{k}_{\perp}$. The Sudakov exponent $S$ describes gluonic radiative corrections not taken into account in the evolution of the wave function. For $\ln \bar{Q} \rightarrow \infty$ it suppresses all contributions to the integral except for those with small quark-antiquark separations. As a consequence the limiting behavior (7) emerges for $\gamma-\pi$ transitions, as we have checked numerically by calculating expression (13) up to $Q^{2}=10^{15} \mathrm{GeV}^{2}$. As $b$ sets the interface between non-perturbative soft gluons contained in the hadronic wave function and perturbative soft gluon contributions resummed in the Sudakov factor, the factorization scale $\mu_{F}$ is taken as $1 / b$. For the renormalization scale we take the 


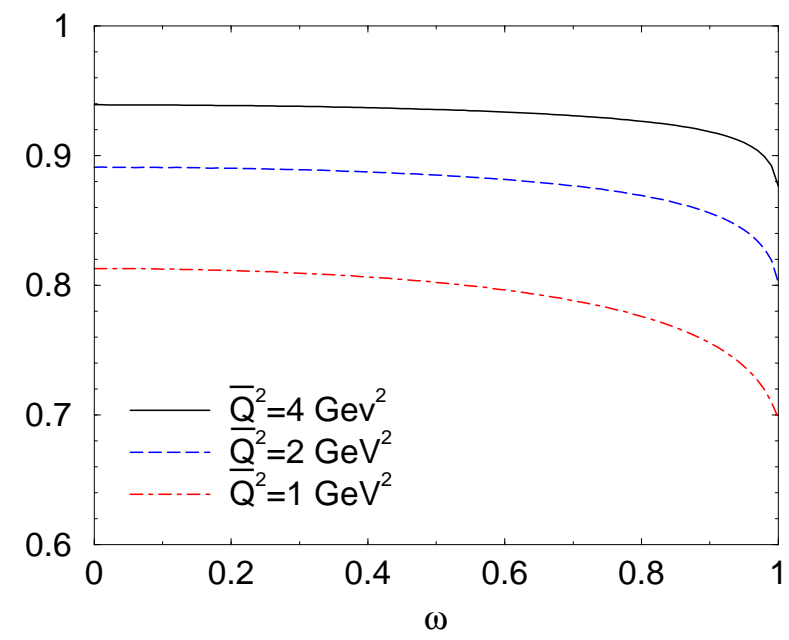

Figure 4: Ratio of $F_{\pi \gamma^{*}}(\bar{Q}, \omega)$ in the modified perturbative approach and in the LO leading twist approximation at $\bar{Q}^{2}=4 \mathrm{GeV}^{2}$ (solid line), $\bar{Q}^{2}=2 \mathrm{GeV}^{2}$ (dashed line), and $\bar{Q}^{2}=1 \mathrm{GeV}^{2}$ (dash-dotted line). Here we have used the wave function (14) in the modified perturbative approach and the asymptotic pion distribution amplitude in the leading twist calculation.

prescription $\mu_{R}=\max \{1 / b, \sqrt{1+\xi \omega} \bar{Q}, \sqrt{1-\xi \omega} \bar{Q}\}$ of [20]. Following [34, 21] we take for the light-cone wave function in $b$-space the simple form

$$
\hat{\Psi}_{\pi}(\xi, \mathbf{b})=\frac{2 \pi f_{\pi}}{\sqrt{6}} \Phi_{\mathrm{AS}}(\xi) \exp \left[-\frac{\pi^{2} f_{\pi}^{2}}{2}\left(1-\xi^{2}\right)\right]
$$

in our estimate. Evaluation of the $\gamma^{*} \gamma \rightarrow \pi$ form factor in the modified perturbative approach using this wave function leads to very good agreement with the CLEO data [7].

In the kinematical range of interest here we find that the Sudakov factor only provides corrections of no more than $1.5 \%$ to $F_{\pi \gamma^{*}}$, and it is thus good enough to retain only the leading logarithmic terms in $S$ as given in [33]. In our kinematics the difference between the asymptotic result (8) and the expression (13) is thus essentially due to the $\mathbf{k}_{\perp}$-corrections to the hard scattering amplitude and not to the perturbative corrections contained in the Sudakov factor. In order to estimate the importance of power corrections we therefore compare (13) with the leading twist result at LO rather than at NLO in $\alpha_{s}$. In Fig. 团 we show the ratio between the form factor evaluated from (14) in the modified perturbative approach and the LO result calculated with $\Phi_{\mathrm{AS}}$ in the leading-twist approximation. As we can see, the corrections are below $10 \%$ for values of $\bar{Q}^{2}=4 \mathrm{GeV}^{2}$, but can go up to $30 \%$ for $\bar{Q}^{2}=1 \mathrm{GeV}^{2}$. As expected, the importance of power corrections decreases as both photons become virtual. This is already signaled by the leading twist result. Indeed, the factor factor $1 /\left(1-\xi^{2} \omega^{2}\right)$ in the convolution (4) controls to which extent $F_{\pi \gamma^{*}}$ is sensitive to contributions from the end-point regions $\xi \rightarrow \pm 1$, where the quark or antiquark in the pion becomes slow and soft effects can become important.

Let us explore how much information on the pion distribution amplitude can be obtained from the $\omega$ dependence of $F_{\pi \gamma^{*}}$ under these circumstances. To this end, we plot in Fig. 5 the form factor for different choices of distribution amplitudes. These have been chosen to give the same value of $\sum_{n} B_{n} \simeq-0.06$ at the scale $\mu_{0}=1 \mathrm{GeV}$, so that up to small corrections they 


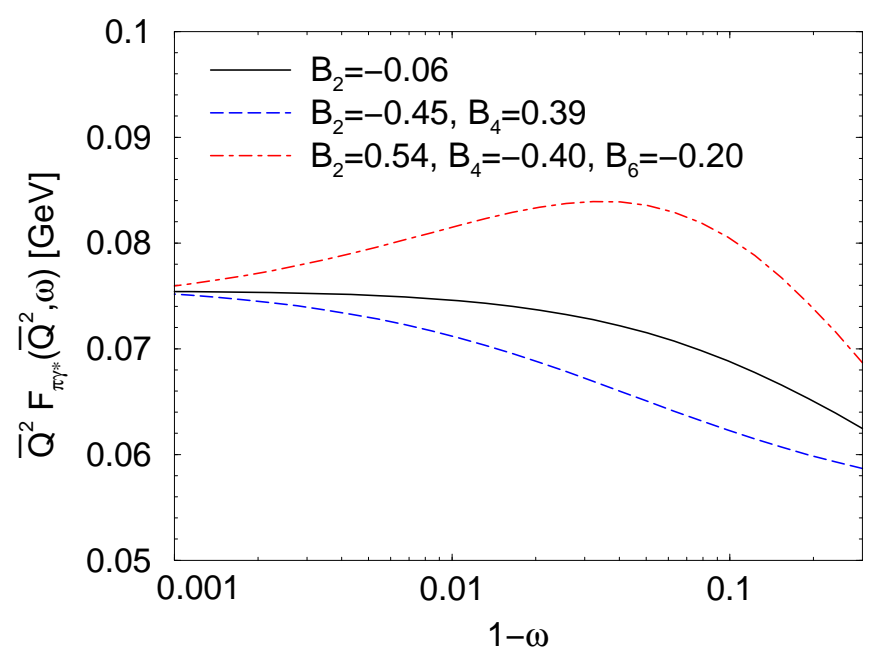

Figure 5: The scaled form factor $\bar{Q}^{2} F_{\pi \gamma^{*}}(\bar{Q}, \omega)$ calculated to NLO in the leading-twist approximation at $\bar{Q}^{2}=4 \mathrm{GeV}^{2}$, using sample distribution amplitudes. The values of the Gegenbauer coefficients are quoted at the scale $\mu_{0}=1 \mathrm{GeV}$. The curves are evaluated from $B_{2}=-0.06$ (solid line), from $B_{2}=-0.45, B_{4}=0.39$ (dashed line) and from $B_{2}=0.54, B_{4}=-0.40$ and $B_{6}=-0.20$ (dash-dotted line). All higher order Gegenbauer coefficients are taken to be zero.

all give the same value of $F_{\pi \gamma}$ in the NLO leading-twist analysis. We see that for Gegenbauer coefficients whose order of magnitude is not implausibly large compared to the theory estimates we have quoted above, one can obtain visible differences in the form factor. In part of the $\omega$ range they can attain $15 \%$ and are thus marginally above the level where we have estimated that at $\bar{Q}^{2}=4 \mathrm{GeV}^{2}$ power corrections can make a reliable extraction of the $B_{n}$ problematic. While one clearly has not enough discriminating power to pin down individual coefficients $B_{n}$, one can gain valid information beyond what can be inferred from real-photon data. Notably, one can check whether the small value of the sum $\sum_{n} B_{n}$, to which $F_{\pi \gamma}$ is mainly sensitive, results from the cancellation of rather large individual terms or from the smallness of the $B_{n}$ themselves. This type of evidence would be rather complementary to the quantitatively more precise information we have argued to be accessible from high $Q^{2}$ data on $F_{\pi \gamma}$.

\section{The region $Q^{\prime 2} \sim Q^{2}$}

As we have seen in Fig. 2, the contribution from Gegenbauer coefficients $B_{n}$ to $F_{\pi \gamma^{*}}$ becomes small as one goes away from $\omega=1$, with a faster rate of decrease for increasing index $n$. To understand this, we observe that the hard scattering kernel in (雨 can be Taylor expanded around $\omega=0$. Using the Gegenbauer expansion (5) of $\Phi_{\pi}(\xi)$, we find the relevant integral to be

$$
\int_{-1}^{1} d \xi \frac{1-\xi^{2}}{1-\xi^{2} \omega^{2}}\left[1+\sum_{n=2,4, \ldots}^{\infty} B_{n} C_{n}^{3 / 2}(\xi)\right]
$$




$$
=\sum_{m=0,2, \ldots}^{\infty} \omega^{m} \int_{-1}^{1} d \xi\left(1-\xi^{2}\right) \xi^{m}+\sum_{\substack{n=2,4, \ldots \\ m=n, n+2, \ldots}}^{\infty} \omega^{m} B_{n} \int_{-1}^{1} d \xi\left(1-\xi^{2}\right) \xi^{m} C_{n}^{3 / 2}(\xi)
$$

for the LO contribution to $F_{\pi \gamma^{*}}$. The condition $m \geq n$ in the sum involving the $B_{n}$ incorporates the orthogonality of Gegenbauer polynomials, as the $\xi$ integrals for $m<n$ are zero. We thus have the remarkable result that in the limit $\omega \rightarrow 0$ a Gegenbauer coefficient $B_{n}$ is suppressed in $F_{\pi \gamma^{*}}$ by a power $\omega^{n}$. This also holds to NLO, as we will show in the appendix, and explains the behavior of the coefficients $c_{n}$ in Fig. 2. Keeping only terms up to $\mathcal{O}\left(\omega^{2}\right)$, we have

$$
\begin{aligned}
F_{\pi \gamma^{*}}(\bar{Q}, \omega)=\frac{\sqrt{2} f_{\pi}}{3 \bar{Q}^{2}} & {\left[1-\frac{\alpha_{s}}{\pi}+\frac{1}{5} \omega^{2}\left(1-\frac{5}{3} \frac{\alpha_{s}}{\pi}\right)\right.} \\
+ & \left.\frac{12}{35} \omega^{2} B_{2}\left(\mu_{F}\right)\left(1+\frac{5}{12} \frac{\alpha_{s}}{\pi}\left\{1-\frac{10}{3} \ln \frac{\bar{Q}^{2}}{\mu_{F}^{2}}\right\}\right)\right]+\mathcal{O}\left(\omega^{4}, \alpha_{s}^{2}\right) .
\end{aligned}
$$

The limiting behavior for $\omega \rightarrow 0$ and to leading order in $\alpha_{s}$ has been derived long ago in [35]. The $\alpha_{s}$-correction to the leading term,

$$
F_{\pi \gamma^{*}}(\bar{Q}, \omega)=\frac{\sqrt{2} f_{\pi}}{3 \bar{Q}^{2}}\left[1-\frac{\alpha_{s}}{\pi}\right]+\mathcal{O}\left(\omega^{2}, \alpha_{s}^{2}\right)
$$

has already been given in [2].

Given the small numerical coefficients in front of $\omega^{2}$, the $\omega$ independent term in Eq. (16) dominates over a rather large range of $\omega$. Even at $\omega \simeq 0.6$ the $\omega^{2}$ corrections amount to less than $15 \%$ if $\left|B_{2}\right|<0.5$. Since higher coefficients $B_{n}$ are suppressed even more strongly, we conclude that in this range of $\omega$ the $\gamma^{*}-\pi$ transition form factor is essentially flat in $\omega$ and independent of the pion distribution amplitude $\Phi_{\pi}$. To illustrate the quality of the small- $\omega$ approximations we compare in Fig. 6 the full result (41) for $F_{\pi \gamma^{*}}$ with the expressions (16) and (17) at $\bar{Q}=2 \mathrm{GeV}$ for a sample distribution amplitude given by $B_{2}=0.54, B_{4}=-0.40$, $B_{6}=-0.20$ at $\mu_{0}=1 \mathrm{GeV}$. The full calculation is in agreement with the CLEO data for $\omega \rightarrow 1$. We see that, although $B_{2}$ in our example is quite large and positive, both approximations are indeed very good for $\omega \lesssim 0.6$.

We thus have a parameter-free prediction of QCD to leading-twist accuracy, which well deserves experimental verification. Any observed deviation from (17) beyond what can reasonably be ascribed to $\mathcal{O}\left(\alpha_{s}^{2}\right)$ terms would be an unambiguous signal for power corrections. Only if the lowest Gegenbauer coefficients $B_{n}$ were extremely large would this conclusion become invalidated, but as we discussed in the previous section, such a scenario could already be ruled out using the region $\omega \simeq 1$. We remark that according to our estimate in Sect. \&, power corrections need not be negligibly small at moderate values of $\bar{Q}$, even for $\omega=0$.

For small $\omega$, the relation (17) has a status comparable to the famous expression of the cross section ratio $R=\sigma\left(e^{+} e^{-} \rightarrow\right.$ hadrons $) / \sigma\left(e^{+} e^{-} \rightarrow \mu^{+} \mu^{-}\right)$. An important difference is however that the hard scale in $R$ is timelike and requires one to stay out of the resonance mass region. In contrast, $F_{\pi \gamma^{*}}$ involves spacelike virtualities and thus offers the possibility to explore the quality of a leading twist approximation down to moderate values of the hard scale. In that respect it is similar to a number of sum rules in inclusive deep inelastic scattering. The fact that (17) should hold in a wide region of $\omega$ raises hope for experimental feasibility of this prediction. 


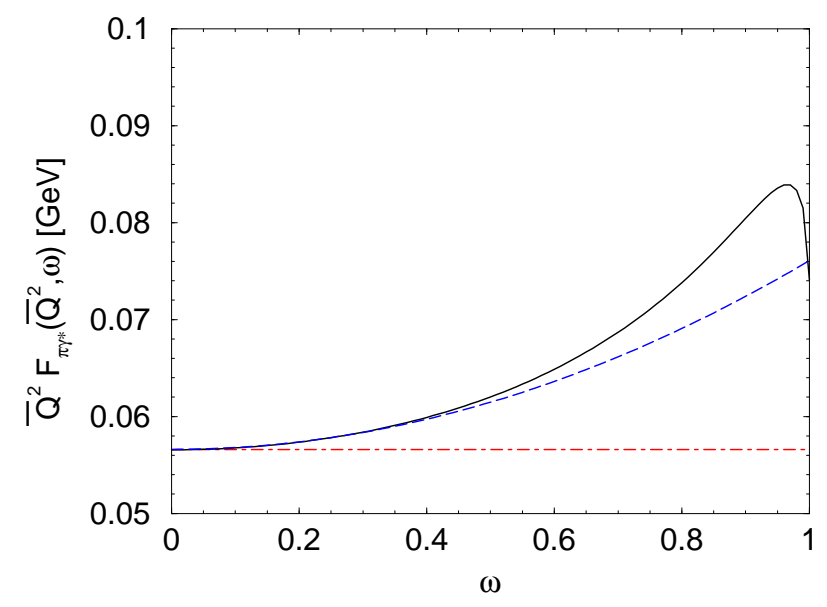

Figure 6: Comparison of a NLO leading twist calculation of the scaled form factor $\bar{Q}^{2} F_{\pi \gamma^{*}}(\bar{Q}, \omega)$ (solid line) with the approximations (16) (dashed line) and (17) (dash-dotted line). The form factor is evaluated with $\mu_{F}=\mu_{R}=\bar{Q}$ at $\bar{Q}=2 \mathrm{GeV}$ for a sample distribution amplitude with $B_{2}=0.54, B_{4}=-0.40, B_{6}=-0.20$, and $B_{n}=0$ for $n \geq 8$. Note the suppressed zero on the $y$ axis.

One may ponder on whether at large $\bar{Q}^{2}$ the form factor $F_{\pi \gamma^{*}}$ has the potential for a determination of $\alpha_{s}$. Our arguments in the appendix suggest that the suppression of $B_{n}$ by $\omega^{n}$ holds to all orders in perturbation theory. Higher orders $\alpha_{s}$ coefficients in (17) for $\Phi_{\pi}=\Phi_{\mathrm{AS}}$ have been obtained in [12] using the conformal operator product expansion. Given phenomenological or theoretical input on $B_{n}$, the $\omega^{n}$ terms could at least be estimated, as could be the size of power corrections. Experimentally, the measurement of $F_{\pi \gamma^{*}}$ should be quite clean. We will however see that truly large $\bar{Q}^{2}$ are not attainable at present facilities because of rather small cross sections.

\section{The $\gamma^{*}-\eta$ and $\gamma^{*}-\eta^{\prime}$ transition form factors}

Let us now discuss the $\gamma^{*}-P$ transition form factors for $P=\eta, \eta^{\prime}$. For the valence Fock states of the mesons we choose a basis with the $\mathrm{SU}(3)_{\mathrm{F}}$ singlet $\left|q \bar{q}^{(1)}\right\rangle=|u \bar{u}+d \bar{d}+s \bar{s}\rangle / \sqrt{3}$ and octet $\left|q \bar{q}^{(8)}\right\rangle=|u \bar{u}+d \bar{d}-2 s \bar{s}\rangle / \sqrt{6}$, and the two-gluon state $|g g\rangle$. This has the advantage that the corresponding distribution amplitudes $\Phi_{P}^{(1)}$ and $\Phi_{P}^{(g)}$ mix under evolution, but $\Phi_{P}^{(8)}$ evolves independently. The solution of the $\mathrm{LO}$ evolution equation for the octet distribution amplitude is given by (5) with Gegenbauer coefficients $B_{P n}^{(8)}$, whereas for the quark singlet and gluon one can write [36]

$$
\begin{aligned}
\Phi_{P}^{(1)}\left(\xi, \mu_{F}\right) & =\Phi_{\mathrm{AS}}(\xi)\left[1+\sum_{n=2,4, \ldots}^{\infty} B_{P n}^{(1)} C_{n}^{3 / 2}(\xi)\right], \\
\Phi_{P}^{(g)}\left(\xi, \mu_{F}\right) & =\frac{\left(1-\xi^{2}\right)^{2}}{16} \sum_{n=2,4, \ldots}^{\infty} B_{P n}^{(g)} C_{n-1}^{5 / 2}(\xi),
\end{aligned}
$$



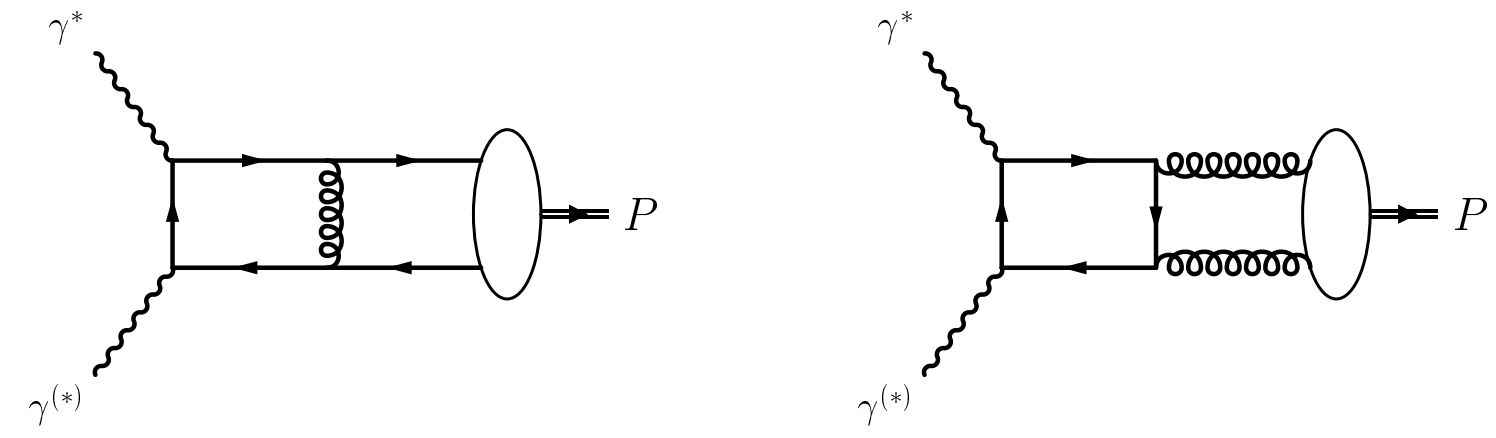

Figure 7: Sample NLO Feynman graphs for the $\gamma^{*} \gamma^{(*)} \rightarrow P$ transition.

with

$$
B_{P n}^{(1)}\left(\mu_{F}\right)=B_{P n}^{(+)}\left(\mu_{F}\right)+B_{P n}^{(-)}\left(\mu_{F}\right), \quad B_{P n}^{(g)}\left(\mu_{F}\right)=a_{n}^{(+)} B_{P n}^{(+)}\left(\mu_{F}\right)+a_{n}^{(-)} B_{P n}^{(-)}\left(\mu_{F}\right),
$$

where the $B_{P n}^{( \pm)}$evolve as in (6) with positive anomalous dimensions $\gamma_{n}^{( \pm)}$. We remark in passing that conflicting results on $\gamma_{n}^{( \pm)}$and $a_{n}^{( \pm)}$are found in the literature [36. Notice that the twogluon distribution amplitude vanishes in the asymptotic limit $\ln \mu_{F} \rightarrow \infty$. It contributes to $F_{P \gamma^{*}}$ only to order $\alpha_{s}$ through the box graph shown in Fig. 7 . The corresponding hard scattering amplitude can be obtained by crossing from the NLO corrections to the Compton amplitude $\gamma^{*} p \rightarrow \gamma^{*} p$, which can be found in [37.

In full analogy to the case of the $\pi$ we can write the $\gamma^{*}-P$ transition form factor as a superposition of Gegenbauer coefficients with $\omega$ dependent weights. In the limit $\omega \rightarrow 1$ one probes essentially the sums $\sum_{n} B_{P n}^{(8)}$ and $\sum_{n} B_{P n}^{(1)}$ of coefficients appearing in the quark distribution amplitudes $\Phi_{P}^{(8)}$ and $\Phi_{P}^{(1)}$. The real-photon limit has been analysed in [5, 8, 38]. Using the mea-

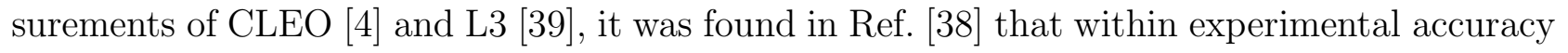
the data on $F_{\eta \gamma}$ and $F_{\eta^{\prime} \gamma}$ is compatible with the asymptotic forms of the quark distribution amplitudes and correspondingly vanishing gluon ones.

When $\omega$ moves away from 1 the form factors become increasingly less sensitive to the higher order coefficients. As in the case of quarks, we find that the Gegenbauer coefficient $B_{P n}^{(g)}$ or the gluon distribution amplitude first appears at order $\omega^{n}$, despite the fact that $\Phi_{P}^{(g)}$ is expanded upon Gegenbauer polynomials $C_{n-1}^{5 / 2}$ instead of $C_{n}^{3 / 2}$. In analogy to (17) we then obtain the prediction

$$
F_{P \gamma^{*}}(\bar{Q}, \omega)=\frac{\sqrt{2} f_{P}^{\mathrm{eff}}}{3 \bar{Q}^{2}}\left[1-\frac{\alpha_{s}}{\pi}\right]+\mathcal{O}\left(\omega^{2}, \alpha_{s}^{2}\right) .
$$

The effective (process-dependent) decay constants are

$$
f_{P}^{\mathrm{eff}}=\frac{f_{P}^{(8)}+2 \sqrt{2} f_{P}^{(1)}}{\sqrt{3}}
$$

where

$$
\left\langle 0\left|J_{5 \mu}^{(i)}\right| P(p)\right\rangle=i f_{P}^{(i)} p_{\mu}, \quad(i=1,8)
$$

are matrix elements of the $\mathrm{SU}(3)_{\mathrm{F}}$ singlet or octet axial vector currents. 
The decomposition of the mesons states $|\eta\rangle$ and $\left|\eta^{\prime}\right\rangle$ on $\left|q \bar{q}^{(1)}\right\rangle,\left|q \bar{q}^{(8)}\right\rangle,|g g\rangle$ and higher Fock states is completely general. It does not refer to $\eta-\eta^{\prime}$ mixing, which is known to occur empirically, and which relates the respective Fock state coefficients for the two mesons. In [40, 41] a quark-flavor mixing scheme has been proposed, which successfully describes many physical processes. In this scheme the physical mesons are obtained from two basis states, $\left|\eta_{q}\right\rangle$ and $\left|\eta_{s}\right\rangle$ by a unitary transformation with a mixing angle $\varphi$. The quark valence Fock states of $\left|\eta_{q}\right\rangle$ and $\left|\eta_{s}\right\rangle$ respectively are $|u \bar{u}+d \bar{d}\rangle / \sqrt{2}$ and $|s \bar{s}\rangle$. This mixing scheme can be justified to the extent that the distribution amplitudes are close to the asymptotic form; evolution does then practically not spoil this scheme. The decay constants (22) are given here by the mixing angle and the two basic decay constants, $f_{q}$ and $f_{s}$. For the effective decay constants above one then finds 40

$$
\begin{aligned}
& f_{\eta}^{\mathrm{eff}}=\frac{5 f_{q} \cos \varphi-\sqrt{2} f_{s} \sin \varphi}{3} \\
& f_{\eta^{\prime}}^{\mathrm{eff}}=\frac{5 f_{q} \sin \varphi+\sqrt{2} f_{s} \cos \varphi}{3}
\end{aligned}
$$

The phenomenological values of the mixing parameters derived in 40 numerically give $f_{\eta}^{\text {eff }}=$ $0.98 f_{\pi}$ and $f_{\eta^{\prime}}^{\text {eff }}=1.62 f_{\pi}$. Similar results are obtained with the large $N_{c}$ values of the $f_{P}^{(i)} 42$. At small $\omega$ and large enough $\bar{Q}^{2}$ the ratio of the $\gamma^{*}-\eta$ and $\gamma^{*}-\eta^{\prime}$ form factors constitutes an accurate measure of the effective decay constants. This can be used for a severe test of the $\eta-\eta^{\prime}$ mixing scheme. As in the pion case, substantial deviations from the small- $\omega$ predictions (20) only occur if there are unexpectedly large power corrections or extremely large Gegenbauer coefficients.

One may extend our analysis to the gluonic transitions $g^{*} g^{*} \rightarrow \eta, \eta^{\prime}$ in the same fashion as we discussed the electromagnetic ones. The case of the $\eta^{\prime}$ has recently been investigated by Ali and Parkhomenko [43, and we can follow their analysis closely. For $\omega \rightarrow 1$ the form factors not only depend on the Gegenbauer coefficients $B_{P, n}^{(1,8)}$ but are also sensitive to variations of the $B_{P n}^{(g)}$, since in contrast to the electromagnetic case both quark and gluon distribution amplitudes now contribute to LO in $\alpha_{s}$. On the other hand, we can still apply our arguments for the suppression of the Gegenbauer coefficients (and hence of the full contribution from $\Phi_{P}^{(g)}$ ) in the limit $\omega \rightarrow 0$. We find for the transition form factors

$$
F_{P g^{*}}(\bar{Q}, \omega)=-\frac{4 \pi \alpha_{s}}{3 \bar{Q}^{2}} C_{P}+\mathcal{O}\left(\omega^{2}, \alpha_{s}^{2}\right)
$$

with effective decay constants

$$
\begin{aligned}
C_{\eta} & =\sqrt{2} f_{q} \cos \varphi-f_{s} \sin \varphi \approx 0.32 f_{\pi}, \\
C_{\eta^{\prime}} & =\sqrt{2} f_{q} \sin \varphi+f_{s} \cos \varphi \approx 1.99 f_{\pi} .
\end{aligned}
$$

The ratio of the two form factors at small $\omega$ is given by $C_{\eta} / C_{\eta^{\prime}}=-\tan \theta_{1}$ which, if measurable, would give access to the badly constrained $\eta-\eta^{\prime}$ mixing parameter $\theta_{1}$ [41. This mixing angle determines the relative decay strength of the $\eta$ and $\eta^{\prime}$ through a weak $\mathrm{SU}(3)_{\mathrm{F}}$ singlet current. Present estimates of the angle $\theta_{1}$ range from $-2^{\circ}$ to $-10^{\circ}$ [41]. 


\begin{tabular}{lcccccc}
\hline \hline & $E_{e^{+}}$ & $E_{e^{-}}$ & $\alpha_{\min }$ & $\pi-\alpha_{\max }$ & $\vartheta_{\min }$ & $\pi-\vartheta_{\max }$ \\
\hline BaBar & 3.1 & 9.0 & 478 & 284 & 667 & 275 \\
Belle & 3.5 & 8.0 & 154 & 112 & 524 & 294 \\
CLEO & 5.3 & 5.3 & 227 & 227 & 314 & 314 \\
\hline \hline
\end{tabular}

Table 1: Minimal and maximal values of polar angles in the laboratory frame imposed by our cuts, as explained in the text. We also give the beam energies for the different experiments. Angles refer to the positron beam axis and are given in mrad, energies are given in $\mathrm{GeV}$.

\section{Cross section estimates}

In order to assess the possibilities of the running experiments BaBar, Belle, and CLEO to investigate $F_{\pi \gamma^{*}}$ we will now estimate cross sections and see how they are affected by acceptance cuts. We first remark that at large $\bar{Q}^{2}$ the cross section for $e^{+} e^{-} \rightarrow e^{+} e^{-} \pi^{0}$ depends on the two photon virtualities roughly like

$$
\frac{d \sigma}{d Q^{2} d Q^{\prime 2}} \sim \frac{1}{Q^{2} Q^{\prime 2}\left(Q^{2}+Q^{\prime 2}\right)^{2}},
$$

where the factors $Q^{2}$ and $Q^{\prime 2}$ are due to the photon flux, and $\left(Q^{2}+Q^{\prime 2}\right)^{2}$ comes from the behavior of $F_{\pi \gamma^{*}}$, which as we have seen behaves like $1 /\left(Q^{2}+Q^{\prime 2}\right)$ with only a mild dependence on $\omega$.

For acceptance cuts we will consider two scenarios. One is that both scattered leptons and the $\pi^{0}$ are seen in the detector. In kinematics where this is not possible because either $Q^{2}$ or $Q^{\prime 2}$ is too small to ensure a sufficiently large lepton scattering angle, one may envisage the detection only of one lepton and the $\pi^{0}$. If experimental resolution permits, the four-momentum of the undetected lepton can then be reconstructed using four-momentum conservation.

We define polar angles with respect to the direction of the positron beam, and take the convention that $Q^{2}$ corresponds to the photon radiated from the $e^{+}$. As typical cuts we impose for tagged leptons a minimum energy of $1 \mathrm{GeV}$ and transverse momentum of $100 \mathrm{MeV}$, and a minimum transverse momentum of $200 \mathrm{MeV}$ for the pion. We further demand that a tagged $e^{+}$ has a polar angle $\alpha_{e^{+}}>\alpha_{\min }$ in the detector. Likewise, we require $\alpha_{e^{-}}<\alpha_{\max }$ for a tagged $e^{-}$, and $\vartheta_{\text {min }}<\vartheta_{\pi}<\vartheta_{\text {max }}$ for the polar angle $\vartheta_{\pi}$ of the pion. For the minimal and maximal angles we take the values given in Table 1. To estimate $F_{\gamma^{*} \pi}$ we use the leading-twist NLO expression (4), taking for simplicity the asymptotic form $\Phi_{\mathrm{AS}}$ of the pion distribution amplitude.

It turns out that the cuts with the most serious impact are the angular restrictions on the scattered leptons, while the precise values of the other cuts have only a mild influence. We show in Fig. 8 the differential $e^{+} e^{-}$cross section in the region $Q^{2} \sim Q^{\prime 2}$. For Belle and CLEO we have imposed detection cuts for both scattered leptons. One clearly sees how for larger $|\omega|$, where at fixed $\bar{Q}^{2}$ one of the photon virtualities becomes small, our cuts do have visible effects. Imposing the same cuts for BaBar leaves essentially no cross section in the kinematical region we are considering. This is due to the limited forward and backward coverage of the BaBar detector: for photon virtualities large enough to bring the scattered lepton within detector acceptance, the cross section is already minute because of its strong decrease (26) with $Q^{2}$ and 

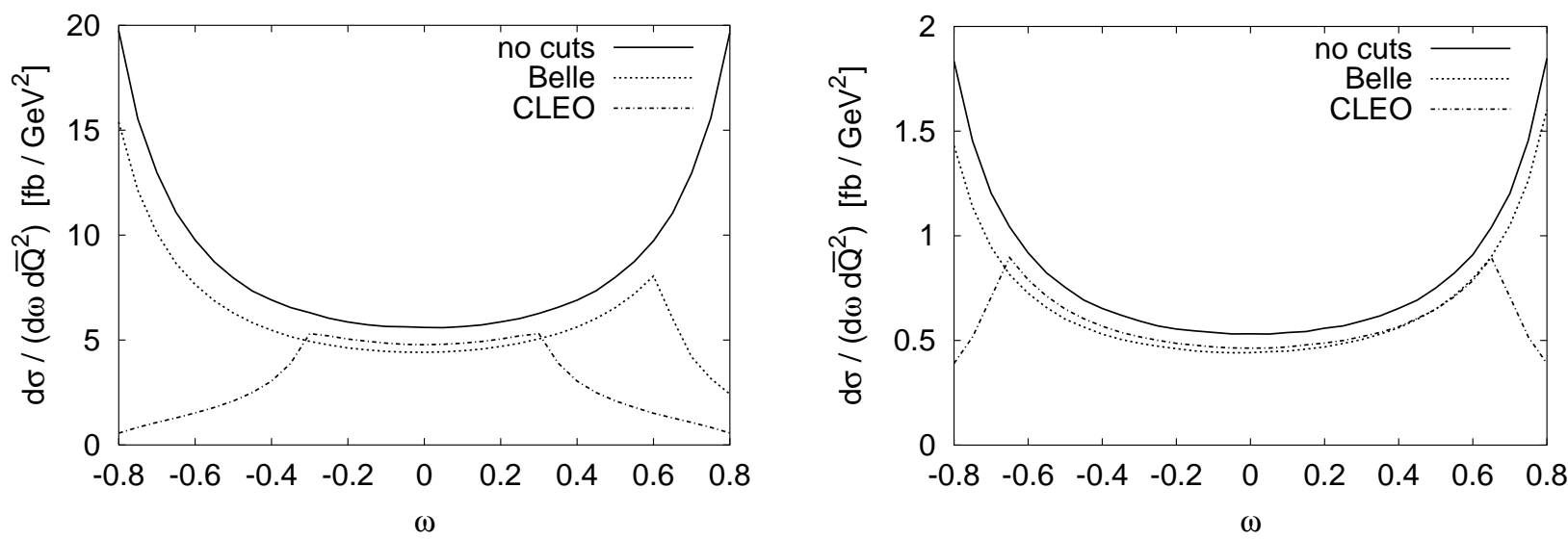

Figure 8: The differential cross section for $e^{+} e^{-} \rightarrow e^{+} e^{-} \pi$ as a function of $\omega$ for $\bar{Q}^{2}=$ $2 \mathrm{GeV}^{2}$ (left) and $\bar{Q}^{2}=4 \mathrm{GeV}^{2}$ (right). Notice that the scaling behavior (26) translates into $d \sigma /\left(d \omega d \bar{Q}^{2}\right) \sim\left(1-\omega^{2}\right)^{-1} \bar{Q}^{-6}$.
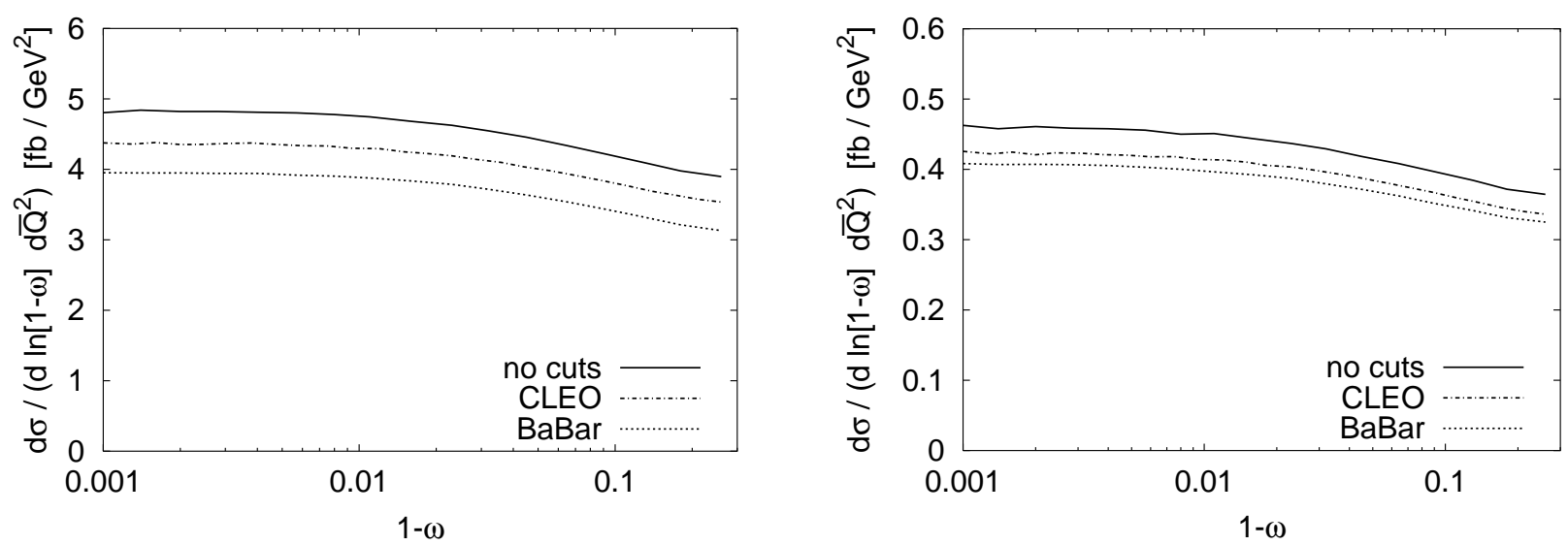

Figure 9: The differential cross section near $\omega=1$ for $\bar{Q}^{2}=2 \mathrm{GeV}^{2}$ (left) and $\bar{Q}^{2}=4 \mathrm{GeV}^{2}$ (right). Corresponding curves with cuts for Belle are between those for BaBar and for CLEO. In this region, the behavior $(26)$ becomes $d \sigma /\left(d \log [1-\omega] d \bar{Q}^{2}\right) \sim \bar{Q}^{-6}$. 
$Q^{\prime 2}$. The same holds if we allow the $e^{+}$to be undetected. Only in the case where the $e^{-}$is untagged do we obtain a signal, not shown in Fig. 8 .

In Fig. 9 we show the differential cross section in the region of $\omega$ close to 1 , requiring only the scattered $e^{+}$and the $\pi^{0}$ to be observed. If instead we require the $e^{-}$and $\pi^{0}$ to be detected in the region of $\omega$ close to -1 , we obtain somewhat smaller cross sections in the case of Belle. For BaBar we have little change at $\bar{Q}^{2}=4 \mathrm{GeV}^{2}$ but almost no signal left at $\bar{Q}^{2}=2 \mathrm{GeV}^{2}$. For CLEO with its symmetric geometry, there is of course no difference between the two cases.

We have also investigated the production of an $\eta$ or $\eta^{\prime}$, imposing the same cuts as described for the pion case. The corresponding cross sections scale approximately like the squared transition form factors $F_{P \gamma^{*}}$, i.e., the mass differences between the $\pi, \eta$, and $\eta^{\prime}$ have only little effect on kinematics and phase space in the region we are investigating.

Concerning the values of cross section estimated here, we recall the benchmark luminosity of $30 \mathrm{fb}^{-1}$ per year of the $B$ factories. With the numbers in Fig. 8 and 9 we conclude that for $\bar{Q}^{2}$ around $2 \mathrm{GeV}^{2}$ studies should be possible, both when $\omega$ is around zero and when $|\omega| \approx 1$. As $\bar{Q}^{2}$ goes up to $4 \mathrm{GeV}^{2}$ and more, event statistics will become increasingly problematic, so that, unfortunately, we do not expect precision measurements to feasible in that region with the current experimental setups. Such studies would greatly benefit from high-luminosity upgrades of the $B$-factories.

We remark that our rate estimates here are restricted to $|\omega| \leq 1-10^{-3}$ and thus do not include the real-photon limit. There the cross section will be much higher and, as emphasized at the end of Sect. 3, measurements with good statistics should be possible at higher values of $Q^{2}$.

\section{Summary}

We have investigated the $\gamma^{*}-\pi$ transition form factor to leading-twist accuracy including $\alpha_{s}$ corrections. The chief purpose of our analysis is to assess what can be learned about the pion distribution amplitude from experimental data on $F_{\pi \gamma^{*}}$.

Our main idea is to use the expansion of $\Phi_{\pi}$ on Gegenbauer polynomials, and to write $F_{\pi \gamma^{*}}$ as a double series in the Gegenbauer coefficients $B_{n}$ and in powers of the variable $\omega$, which describes the difference of the two photon virtualities. We find that, contrary to what one may expect, it is very difficult to obtain information on the $B_{n}$ beyond what can be inferred from the case where one of the photons is quasi-real, which essentially constrains the sum of Gegenbauer coefficients. Only for $\omega$ values close to but less than 1 can one get more information. Effects on $F_{\pi \gamma^{*}}$ of the order of $10 \%$ to $15 \%$ can be obtained using coefficients whose magnitude is not implausibly large compared to theory estimates. Data in that range of $\omega$ can, for instance, allow a check whether the small value of the sum $\sum_{n} B_{n}$, extracted from the CLEO real-photon data, results from cancellations of rather large individual terms or from the smallness of the $B_{n}$ themselves. This type of information would be a valuable input into other phenomenological studies of $\Phi_{\pi}$.

One of our main findings is that a Gegenbauer coefficient $B_{n}$ in $\Phi_{\pi}$ contributes to $F_{\pi \gamma^{*}}$ with a weight proportional to $\omega^{n}$. For a large range of $\omega$, in fact for $\omega \lesssim 0.6$, we find that the form factor is independent of the Gegenbauer coefficients to a high degree of accuracy. Although this is bad news for a determination of $\Phi_{\pi}$, it entails a parameter free QCD prediction of the 
$\gamma^{*} \gamma^{*} \rightarrow \pi$ form factor. Any clear deviation from this result observed in experiment would be an unambiguous signal for power corrections, provided that the lowest Gegenbauer coefficients $B_{n}$ are not extremely large, a scenario which could be ruled out using the region $\omega \simeq 1$. In a wide region of $\omega$ around zero, data on $F_{\pi \gamma^{*}}$ would thus permit one to test the quality of leading twist QCD in the spacelike region, in a similar fashion as sum rules in deep inelastic scattering.

Analogous results hold for the $\gamma^{*} \gamma^{*} \rightarrow \eta$ and $\gamma^{*} \gamma^{*} \rightarrow \eta^{\prime}$ form factors. Note that here the gluon distribution amplitudes contribute indirectly through mixing in the evolution and directly to order $\alpha_{s}$. Their contribution is further suppressed for small $\omega$. Data on $F_{\eta \gamma^{*}}$ and $F_{\eta^{\prime} \gamma^{*}}$ in that region could rather cleanly determine of a linear combination of the flavor singlet and octet decay constants of these mesons, and thus give valuable information on $\eta-\eta^{\prime}$ mixing.

Cross section estimates of the process $e^{+} e^{-} \rightarrow e^{+} e^{-} \pi^{0}$ at the running experiments BaBar, Belle, and CLEO indicate that it should be possible, although challenging, to measure the transition form factors for virtual photons up to about $\bar{Q}^{2} \lesssim 4 \mathrm{GeV}^{2}$. Limiting factors for these measurements are luminosity and the acceptance for lepton detection in the forward and backward regions of the detector. Measurements with better statistics and at higher $\bar{Q}^{2}$ might be feasible at high-luminosity upgrades of the present $B$-factories.

Concerning the real-photon limit, we argue that although the present data on $F_{P \gamma}$ favor a small value for the sum $\sum_{n} B_{n}$, more precise statements can only be made at the price of theory assumptions on the nature and size of power corrections to the leading-twist result. High statistics measurements at large virtualities $Q^{2}$ would greatly alleviate this problem and should be feasible at BaBar, Belle, and CLEO. They should be worthwhile, since the pseudoscalar meson distribution amplitudes are fundamental quantities describing meson structure and providing benchmark tests for nonperturbative methods in QCD. They are also an input required for the calculation of several phenomenologically important processes in hard-scattering approaches. An example are exclusive nonleptonic $B$ meson decays into pseudoscalars, where a good understanding of the strong interaction dynamics would enhance the prospects of extracting information on $C P$ violation.

\section{Acknowledgments}

We wish to acknowledge discussion with A. Ali, J. C. Collins, T. Feldmann, A. Grozin, R. Jakob, A. Khodjamirian, H. Koch, D. Müller and V. Savinov. C. V. thanks the Deutsche Forschungsgemeinschaft for support.

\section{Appendix}

The NLO hard scattering kernel in (幽), evaluated in the $\overline{\mathrm{MS}}$ scheme, reads

$$
\begin{aligned}
\mathcal{K}= & \frac{1}{6}[(1+\xi \omega) \ln (1-\xi \omega)+4(1-\omega) \ln (1-\omega) \\
& \left.+(1+\xi \omega) \ln ^{2}(1-\xi \omega)-(1-\omega) \ln ^{2}(1-\omega)-9(1+\xi \omega)\right] \\
& +\frac{1}{6} \ln \frac{\bar{Q}^{2}}{\mu_{F}^{2}}[2(1+\xi \omega) \ln (1-\xi \omega)-2(1-\omega) \ln (1-\omega)+3(1+\xi \omega)]
\end{aligned}
$$




$$
\begin{aligned}
& +\frac{1}{6 \omega^{2}\left(1-\xi^{2}\right)}\left[2(1+\xi \omega)\left(1+\xi \omega-2 \omega^{2}\right) \ln (1-\xi \omega)-2(1+\omega)\left(1+\omega-2 \omega^{2}\right) \ln (1-\omega)\right. \\
& \left.\quad-(1+\xi \omega)\left(1-\omega^{2}\right) \ln ^{2}(1-\xi \omega)+(1+\omega)\left(1-\omega^{2}\right) \ln ^{2}(1-\omega)\right] \\
& +\frac{1-\omega^{2}}{3 \omega^{2}\left(1-\xi^{2}\right)} \ln \frac{\bar{Q}^{2}}{\mu_{F}^{2}}[(1+\xi \omega) \ln (1-\xi \omega)-(1+\omega) \ln (1-\omega)] \\
& +\{\omega \rightarrow-\omega\} .
\end{aligned}
$$

We will now show that the relevant convolution in the NLO part of $F_{\pi \gamma^{*}}$ can be written as

$$
\begin{aligned}
& \int_{-1}^{1} d \xi \frac{1-\xi^{2}}{1-\xi^{2} \omega^{2}} \mathcal{K}(\omega, \xi)\left[1+\sum_{n=2,4, \ldots}^{\infty} B_{n} C_{n}^{3 / 2}(\xi)\right] \\
& \quad=\sum_{m=0,2, \ldots}^{\infty} \omega^{m} \int_{-1}^{1} d \xi\left(1-\xi^{2}\right) p_{m}(\xi)+\sum_{\substack{n=2,4, \ldots \\
m=n, n+2, \ldots}}^{\infty} \omega^{m} B_{n} \int_{-1}^{1} d \xi\left(1-\xi^{2}\right) p_{m}(\xi) C_{n}^{3 / 2}(\xi),
\end{aligned}
$$

where the $p_{m}(\xi)$ are polynomials in $\xi$ of order $m$. Due to the orthogonality of the $C_{n}^{3 / 2}(\xi)$ the sum involving the $B_{n}$ is here again restricted to $m \geq n$. To show (28) it is enough to establish that $\mathcal{K}(\omega, \xi)$ can be expanded in a double Taylor series

$$
\sum_{m=0}^{\infty} \sum_{l=0}^{m} d_{m-l, l} \omega^{2 m} \xi^{2 l}=\sum_{k, l=0}^{\infty} d_{k, l} \omega^{2 k}(\omega \xi)^{2 l},
$$

i.e., that $\mathcal{K}$ is analytic in the two variables $\omega^{2}$ and $\omega^{2} \xi^{2}$ at $\omega^{2}=\omega^{2} \xi^{2}=0$. One readily sees that $\mathcal{K}$ is even in $\omega$ and $\xi$, which is a consequence of Bose symmetry and charge conjugation invariance. Further inspection of (27) shows that we can write

$$
\mathcal{K}=f\left(\omega^{2}, \omega^{2} \xi^{2}\right)+\frac{g\left(\omega^{2}, \omega^{2} \xi^{2}\right)}{\omega^{2}-\omega^{2} \xi^{2}}
$$

where $f$ and $g$ are analytic in their variables around $\omega^{2}=\omega^{2} \xi^{2}=0$. Hence $g$ is also analytic in $\omega^{2}$ and $\omega^{2}-\omega^{2} \xi^{2}$. Finally one can see from (27) that $g$ is zero for $\xi=1$. The apparent pole at $\omega^{2}-\omega^{2} \xi^{2}=0$ thus cancels in (30), and $g /\left(\omega^{2}-\omega^{2} \xi^{2}\right)$ also has the required analyticity properties.

It is not surprising that $\mathcal{K}$ is analytic in the pair of variables $\omega$ and $\omega \xi$. Apart from the appropriate subtraction of collinear singularities, the NLO hard scattering kernel is the amplitude for the partonic subprocess $\gamma^{*}(q)+\gamma^{*}\left(q^{\prime}\right) \rightarrow q(k)+\bar{q}\left(k^{\prime}\right)$ in the collinear limit, i.e., at $\left(k+k^{\prime}\right)^{2}=0$. From the relations $q^{2}=-\bar{Q}^{2}(1-\omega)$ and $\left(q^{\prime}-k^{\prime}\right)^{2}=-\bar{Q}^{2}(1-\omega \xi)$ we see that for $\bar{Q}^{2}>0$ analyticity in $\omega$ and $\omega \xi$ around $\omega=\omega \xi=0$ is equivalent to analyticity in the spacelike invariants $q^{2}$ and $\left(q^{\prime}-k^{\prime}\right)^{2}$. This suggests that our result (15) will generalize to higher orders in $\alpha_{s}$, provided appropriate analyticity properties of the collinear subtraction terms in the hard scattering kernel.

\section{References}

[1] G.P. Lepage and S.J. Brodsky, Phys. Rev. D 22, 2157 (1980). 
[2] F. Del Aguila and M.K. Chase, Nucl. Phys. B 193, 517 (1981).

[3] E. Braaten, Phys. Rev. D 28, 524 (1983);

E.P. Kadantseva, S.V. Mikhailov and A.V. Radyushkin, Sov. Jour. Nucl. Phys. 44, 326 (1986).

[4] J. Gronberg et al., CLEO collaboration, Phys. Rev. D 57, 33 (1998) hep-ex/9707031.

[5] R. Jakob, P. Kroll and M. Raulfs, Jour. Phys. G 22, 45 (1996) hep-ph/9410304.

[6] S. Ong, Phys. Rev. D 52, 3111 (1995).

[7] P. Kroll and M. Raulfs, Phys. Lett. B 387, 848 (1996) hep-ph/9605264.

[8] F.-G. Cao, T. Huang and B.-Q. Ma, Phys. Rev. D 53, 6582 (1996) [hep-ph/9603330;

V.V. Anisovich, D.I. Melikhov and V.A. Nikonov, Phys. Rev. D 55, 2918 (1997) hepph/9607215.

[9] A. Anselm, A. Johansen, E. Leader and Ł. Łukaszuk, Z. Phys. A 359, 457 (1997) hep$\mathrm{ph} / 9603444$.

[10] I.V. Musatov and A.V. Radyushkin, Phys. Rev. D 56, 2713 (1997) hep-ph/9702443.

[11] S.J. Brodsky, C.-R. Ji, A. Pang and D.G. Robertson, Phys. Rev. D 57, 245 (1998) hepph/9705221].

[12] D. Müller, Phys. Rev. D 58, 054005 (1998) hep-ph/9704406.

[13] B. Melić, B. Nižić and K. Passek, hep-ph/0107295.

[14] P. Kessler and S. Ong, Phys. Rev. D 48, 944 (1993).

[15] C. Caso et al. [Particle Data Group Collaboration], Eur. Phys. J. C 3, 1 (1998).

[16] D. Müller, Phys. Rev. D 51, 3855 (1995) hep-ph/9411338.

[17] J. Bolz, P. Kroll and G.A. Schuler, Phys. Lett. B 392, 198 (1997) hep-ph/9610265; Eur. Phys. J. C 2, 705 (1998) hep-ph/9704378.

[18] V.M. Braun, A. Khodjamirian and M. Maul, Phys. Rev. D 61, 073004 (2000) hepph/9907495].

[19] N.G. Stefanis, W. Schroers and H.C. Kim, Eur. Phys. J. C 18, 137 (2000) hepph/0005218].

[20] H.-N. Li and G. Sterman, Nucl. Phys. B 381, 129 (1992).

[21] R. Jakob and P. Kroll, Phys. Lett. B 315, 463 (1993) hep-ph/9306259.

[22] G. Bertsch, S.J. Brodsky, A.S. Goldhaber and J. F. Gunion, Phys. Rev. Lett. 47, 297 (1981). 
[23] N.N. Nikolaev, W. Schäfer and G. Schwiete, Phys. Rev. D 63, 014020 (2001) hepph/0009038;

L. Frankfurt, G.A. Miller and M. Strikman, hep-ph/0010297;

V.M. Braun, D.Y. Ivanov, A. Schäfer and L. Szymanowski, Phys. Lett. B 509, 43 (2001) hep-ph/0103275;

V. Chernyak, hep-ph/0103295.

[24] A. Khodjamirian, Eur. Phys. J. C 6, 477 (1999) hep-ph/9712451.

[25] T. Yeh, hep-ph/0107018.

[26] A. Schmedding and O. Yakovlev, Phys. Rev. D 62, 116002 (2000) hep-ph/9905392.

[27] A. Khodjamirian, Nucl. Phys. B 605, 558 (2001) hep-ph/0012271.

[28] V.M. Braun and I.E. Filyanov, Z. Phys. C 44, 157 (1989).

[29] L. Del Debbio, M. Di Pierro, A. Dougall and C. Sachrajda [UKQCD collaboration], Nucl. Phys. Proc. Suppl. 83, 235 (2000) hep-lat/9909147.

[30] A.P. Bakulev, S.V. Mikhailov and N.G. Stefanis, Phys. Lett. B 508, 279 (2001) hep$\mathrm{ph} / 0103119$.

[31] S. Dalley, Phys. Rev. D 64, 036006 (2001) hep-ph/0101318];

M. Burkardt and S.K. Seal, hep-ph/0102245.

[32] V.Yu. Petrov, M.V. Polyakov, R. Ruskov, C. Weiss and K. Goeke, Phys. Rev. D 59, 114018 (1999) hep-ph/9807229;

M. Praszalowicz and A. Rostworowski, hep-ph/0105188.

[33] J. Botts and G. Sterman, Nucl. Phys. B 325, 62 (1989).

[34] G.P. Lepage, S.J. Brodsky, T. Huang and P.B. Mackenzie, in Banff Summer Insitute 1981, Particles and Fields 2, ed. by A.Z. Capri and A.N. Kamal (Plenum Press, New York 1983), p 83.

[35] J.M. Cornwall, Phys. Rev. Lett. 16, 1174 (1966);

G. Köpp, T.F. Walsh and P. Zerwas, Nucl. Phys. B 70, 461 (1974);

V.A. Novikov, M.A. Shifman, A.I. Vainshtein, M.B. Voloshin and V.I. Zakharov, Nucl. Phys. B 237, 525 (1984).

[36] Th. Ohrndorf, Nucl. Phys. B 186, 153 (1981);

M.A. Shifman and M.I. Vysotsky, Nucl. Phys. B 186, 475 (1981);

V.N. Baier and A.G. Grozin, Nucl. Phys. B 192, 476 (1981).

[37] L. Mankiewicz, G. Piller, E. Stein, M. Vänttinen, T. Weigl, Phys. Lett. B 425, 186 (1998) [hep-ph/9712251];

X. Ji and J. Osborne, Phys. Rev. D 58, 094018 (1998) hep-ph/9801260.

[38] T. Feldmann and P. Kroll, Eur. Phys. J. C 5, 327 (1998) hep-ph/9711231. 
[39] M. Acciarri et al. [L3 Collaboration], Phys. Lett. B 418, 399 (1998).

[40] Th. Feldmann, P. Kroll and B. Stech, Phys. Rev. D 58, 1140006 (1998) hep-ph/9802409.

[41] Th. Feldmann, P. Kroll and B. Stech, Phys. Lett. B 449, 339 (1999) hep-ph/9812269.

[42] R. Kaiser and H. Leutwyler, hep-ph/9806336.

[43] A. Ali and A.Ya. Parkhomenko, hep-ph/0012212. 